\title{
Role of the Olivo-Cerebellar System in Timing
}

\author{
Duo Xu, ${ }^{1}$ Tao Liu, ${ }^{2}$ James Ashe, ${ }^{1,2}$ and Khalafalla O. Bushara ${ }^{1,3}$ \\ ${ }^{1}$ Neurology Service and ${ }^{2}$ Brain Science Center, Minneapolis Veterans Affairs Medical Center, Minneapolis, Minnesota 55417, and ${ }^{3}$ University of Minnesota \\ Ataxia Center, Minneapolis, Minnesota 55455
}

Timing has been proposed as a basic function of the cerebellar cortex (particularly the climbing fiber afferents and their sole source, the inferior olive) that explains the contribution of the cerebellum to both motor control and nonmotor cognitive functions. However, whether the olivo-cerebellar system mediates time perception without motor behavior remains controversial. We used event-related functional magnetic resonance imaging to dissociate the neural correlates of the perceptual from the motor aspects of timing. The results show activation of multiple areas within the cerebellar cortex during both perception and motor performance of temporal sequences. The results further show that the inferior olive was activated only when subjects perceived the temporal sequences without motor activity. This finding is most consistent with electrophysiological studies showing decreased responsiveness of the inferior olivary neurons to sensory input during expected, self-produced movement. Our results suggest that the primary role of the inferior olive and the climbing fiber system in timing is the encoding of temporal information independent of motor behavior.

Key words: fMRI; motor; perception; temporal; inferior olive; cerebellum

\section{Introduction}

In addition to its traditional role in motor coordination, the cerebellum has been implicated in diverse perceptual and higher cognitive functions (Petersen et al., 1988; Leiner et al., 1993; Kim et al., 1994; Gao et al., 1996; Allen et al., 1997; Schmahmann and Sherman, 1998). How the cerebellum contributes to this multitude of behavioral tasks remains unknown. The remarkably homogeneous and repetitive microstructure and circuitry throughout the cerebellar cortex suggest that the cerebellum performs the same basic function or general computation that is shared by both motor and nonmotor cognitive processes (Thach, 1997; Schmahmann, 2004). This has been the basis for several theories encompassing the nonmotor functions of the cerebellum (Keele and Ivry, 1990; Ito, 1993; Schmahmann, 2004). One proposal is that the cerebellum mediates the acquisition and retention of learned behavior including movements and thoughts (Ito, 1993). Others propose that the cerebellum performs a universal transform by which mismatches between intended and perceived outcomes of motor and nonmotor cognitive processes are monitored and corrected (Oscarsson, 1980; Schmahmann, 2004). Another hypothesis is that the cerebellum functions as an internal timing device in the milliseconds range for both motor and nonmotor processes (Keele and Ivry, 1990). A timekeeping or "clock" function has long been postulated for the cerebellar cortex and the inferior olive (the sole source of climbing fiber inputs to Purkinje cells) based on their unique microstructure and intrinsic rhythmic oscillatory properties (Braitenberg, 1967; Llinas and

Received 0ct. 8, 2005; revised April 23, 2006; accepted April 24, 2006

This work was supported by the Department of Veterans Affairs, Biotechnology Research Resource (Grant P41 008079), and the MIND Institute.

Correspondence should be addressed to Dr. Khalafalla 0. Bushara, Neurology Service (127), Minneapolis Veterans Affairs Medical Center, One Veterans Drive, Minneapolis, MN 55417. E-mail: busha001@umn.edu. DOI:10.1523/JNEUROSCI.0038-06.2006

Copyright $\odot 2006$ Society for Neuroscience $\quad$ 0270-6474/06/265990-06\$15.00/0
Sasaki, 1989; De Zeeuw et al., 1998). However, the primary role of the inferior olive and the climbing fiber system in timing and motor control is poorly understood and remains a matter of debate. One view is that the role of the olivo-cerebellar system in timing and error correction of ongoing movement is limited given the relatively slow intrinsic firing rate of the olivary neurons and their variable responsiveness to active movement (Thach, 1968; Armstrong, 1974; Keating and Thach, 1995; Horn et al., 1996; Gibson et al., 2004). However, it has also been suggested that timing of fast movement and modulation of cerebellar output during movement is accomplished by rhythmic and synchronized firing of ensembles of olivary neurons and their population code rather than individual neuronal oscillations (Welsh et al., 1995; Welsh, 2002). Welsh et al. (2005) further hypothesized that disorders such as autism may be explained by inferior olive dysfunction resulting in impairment of temporal processing and reaction to fast sensory input.

At the system level, human lesion studies localized cerebellar timing mechanisms to the cerebellar cortex and suggested a medial/lateral dissociation (Ivry et al., 1988). Medial lesions impaired timing by causing variability of the motor/implementation component of a finger-tapping task, whereas lateral lesions caused clock variability suggesting that the lateral cerebellar hemispheres are critical for the internal timing system (Ivry et al., 1988). However, other studies questioned this medial/lateral dissociation and suggested a superior/inferior dissociation with impairment of both time perception and motor timing in patients with superior cerebellar lesions (Harrington et al., 2004a).

Similar to lesion studies, functional imaging studies implicated multiple regions of the cerebellar cortex and showed activation of both the medial and lateral zones during timing tasks (Ramnani and Passingham, 2001; Harrington et al., 2004b). However, the cerebellar responses specific to the perceptual and motor aspects of timing have been difficult to dissociate 
(Schubotz et al., 2000; Harrington et al., 2004b). Whether the olivo-cerebellar system mediates time perception without motor behavior remains controversial (Rao et al., 2001). The aim of this study was to dissociate time perception per se from the production of timed movements using event-related functional magnetic resonance imaging (fMRI), focusing on the cerebellar system.

\section{Materials and Methods}

Subjects. Twelve healthy volunteers (six men and six women; age, 18-40 years; mean \pm SD, $22 \pm 2.7$ years) participated in the study. All subjects were right-handed and had normal or corrected-to-normal visual acuity. All subjects gave a written informed consent according to the guidelines approved by the Minneapolis Veterans Affairs Medical Center and the University of Minnesota human subjects committees.

Behavioral task. fMRI scans were obtained while subjects performed multiple timing tasks requiring perception and motor performance of complex temporal sequences of visual stimuli (rhythms) in the hundreds-of-milliseconds range (Fraisse, 1974). Visual stimulation was used to avoid MRI noise interference with auditory stimuli. To effectively identify the neural responses specific to perceptual and motor timing, we used multiple timing tasks with variable event duration and interevent intervals. Rhythms consisted of a sequence of six visual stimuli (200 ms stimulus duration) presented within 4,6 , or $8 \mathrm{~s}$. A combination of two interstimulus intervals (related with 1:2, 1:3, 1:4, or 1:5 ratio) were selected from eight intervals $(200,400,600,800,1000,1200,1600$, and 2400 $\mathrm{ms}$ ) to construct metrical rhythms that are more precisely reproduced than nonmetrical rhythms (rhythms related with non-integer ratios; e.g., 1:2.5) (Essens and Povel, 1985).

The experiment was designed for both subtraction analysis and cognitive conjunction analysis by which areas specific to a cognitive process shared by different behavioral tasks are identified as those activated in common to these tasks and without significant interaction between the tasks (Price and Friston, 1997).

Subjects performed three different tasks: (1) stimulus-guided synchronized movement with the rhythm by pushing a button "on-thebeat" with the right index finger (SYNCHRONIZE); (2) perception and reproduction of rhythms in which subjects observed the rhythms and, after a variable delay, reproduced the rhythms from memory by pushing the button (REPRODUCE); and (3) delayed matching task in which subjects observed a rhythm and, after a variable delay, observed a second (comparison) rhythm and indicated whether the two rhythms were the same by pushing a button once with the index finger (MATCH). In the motor response phase in REPRODUCE trials, the visual stimulus appeared with each button press, therefore, except for stimulus/response order (i.e., whether motor response was stimulus-guided or reproduced from memory), the motor response phase in both the SYNCHRONIZE and REPRODUCE trials required production of timed movements. Similarly, the perception phase in both the REPRODUCE and MATCH trials required perception of temporal sequences and differed only in whether a motor response was to be performed after the delay (no motor preparation occurred after rhythm perception in the MATCH trials). Thus, rhythm perception was common to both the REPRODUCE and MATCH trials, whereas motor performance of rhythm was common to both the SYNCHRONIZE and REPRODUCE trials. The use of multiple timing tasks allowed for cognitive conjunction analysis to identify the neural responses to the perception and motor performance of rhythm, regardless of the task performed (REPRODUCE or MATCH for rhythm perception and SYNCHRONIZE or REPRODUCE for motor performance of rhythm) (Price and Friston, 1997)

To control for visual stimulation and motor response, subjects performed control trials in which rhythms were isochronous (with equal interstimulus intervals of 467,800 , or $1133 \mathrm{~ms}$ for 4,6 , and 8 s sequences, respectively). The number of stimuli in control trials was identical to that presented in the SYNCHRONIZE, REPRODUCE, and MATCH trials. Thus, the SYNCHRONIZE, REPRODUCE, and MATCH trials differed from the corresponding control trials only in the complexity of the temporal structure of the rhythms. Therefore, responses specific to percep- tion and motor performance of rhythm could appropriately be identified using cognitive subtraction methods.

During scanning, subjects performed three runs (each with an equal number of SYNCHRONIZE and REPRODUCE trials) and three runs (each with equal number of REPRODUCE and MATCH trials). Twelve to 14 test trials with complex rhythms were presented in each run. Twelve to 14 different complex rhythms were used in a run to minimize familiarity with the complex rhythms. These were chosen from 15 complex rhythms with the following interstimulus interval combinations: EAAEAA, CCACCA, AEAAEA, ACCACC, AAEAAE, CACCAC, EAAAEA, CCFCCF, GBBGBB, BGBBGB, HCCHCA, CHCCHC, DDGDDG, GDDGDF, and CCHCCH. The interstimulus intervals were as follows: $\mathrm{A}=200 \mathrm{~ms}, \mathrm{~B}=400 \mathrm{~ms}, \mathrm{C}=600 \mathrm{~ms}, \mathrm{D}=800 \mathrm{~ms}, \mathrm{E}=1000 \mathrm{~ms}$, $F=1200, \mathrm{G}=1600$, and $\mathrm{H}=2400 \mathrm{~ms}$. An equal number of control trials (12-14 trials) were presented in each run. Control rhythms had fixed interstimulus intervals as follows: IIIII, DDDDDD, and JJJJJJ, where I = $467 \mathrm{~ms}, \mathrm{D}=800 \mathrm{~ms}$, and $\mathrm{J}=1133 \mathrm{~ms}$. Tasks and trial types within each run were presented in pseudorandom order, counterbalanced across subjects. Each run was 6 min long. The intertrial interval was varied between 4 and $16 \mathrm{~s}$ [average, $4 \mathrm{~s}$ (2TR) excluding task cues]. This jittered design was used to improve estimation power of event-related activity (Dale, 1999).

The visual display was projected through a back-lit screen at the head of the scanner bed and viewed via a mirror attached to the head coil. Subjects viewed a circle (visual angle, $1.2^{\circ}$ ) at the center of a square frame (visual angle, $4.7^{\circ}$ ) on a black background. Before each task, a written cue ("Synchronize," "Reproduce," or "Match") was presented for $2 \mathrm{~s}$. The square frame brightened to signal the start and end of each rhythm duration. Subjects practiced for one to two runs before scanning.

$f M R I$. Blood oxygenation level-dependent contrast functional images were acquired with a 3 T MRI scanner (Magnetom Trio; Siemens, Erlangen, Germany) using a gradient echoplanar $\left(\mathrm{T} 2^{*}\right)$ sequence with the following parameters: echo time, $30 \mathrm{~ms}$; repeat time, $2000 \mathrm{~ms}$; flip angle, $90^{\circ}$; field of view, $192 \times 192$; in-plane resolution, $3 \times 3 \mathrm{~mm}$; slice thickness, $3 \mathrm{~mm}$ ). Thirty-four axial slices covering the entire brain, cerebellum, and brain stem were obtained. A high-resolution anatomical T1 image was obtained with the following parameters: echo time, $4.7 \mathrm{~ms}$; repeat time, $20 \mathrm{~ms}$; flip angle, $22^{\circ}$; field of view, $256 \times 256$; in-plane resolution, $1 \times 1 \mathrm{~mm}$; slice thickness, $1 \mathrm{~mm}$ ).

Data analysis. fMRI data were analyzed using Statistical Parametric Mapping (SPM2) software (Wellcome Department of Cognitive Neurology, London, UK; http://www.fil.ion.ucl.ac.uk/spm) implemented in MATLAB (Mathworks, Sherborn, MA). The first three volumes of each run were discarded to allow for T1 equilibration. The remaining 177 volumes in each run were realigned to the first image, sinc-interpolated over time to correct for phase advance during volume acquisition, and normalized to the Montreal Neurological Institute (MNI) brain template with an enlarged box to include the entire cerebellum and brain stem $(Z=85$ to $-70 \mathrm{~mm})$. T1-weighted anatomical images were coregistered to the functional scans and transformed into the same MNI template. The functional data were spatially smoothed with a Gaussian kernel of 8 $\mathrm{mm}$ full-width at half-maximum to decrease spatial noise. The head motion parameters were added as covariates of no interest in the statistical analysis model.

Statistical analysis was performed in two levels of a mixed effects model. In the first-level analysis (fixed effects), a canonical hemodynamic response function was used as a covariate in a general linear model as implemented in SPM2 yielding a parameter estimate for each event for each voxel. Seven events were modeled for each subject: motor response for SYNCHRONIZE trials; perception, delay, and motor response for REPRODUCE trials; first-rhythm perception, delay, and second (comparison)-rhythm perception for MATCH trials. Identical events were modeled for control trials. Perception of second-rhythm and motor response in MATCH trials and task cues were explicitly modeled and removed. The remaining intertrial interval represents the baseline. Additional statistical comparisons were limited to four events of interest: (1) motor response in the SYNCHRONIZE trials; (2) rhythm perception in the REPRODUCE trials; (3) motor response in the REPRODUCE trials; and (4) perception of first rhythm in the MATCH trials. Statistical para- 
Table 1. Event-related activations of timing tasks

\begin{tabular}{|c|c|c|}
\hline Region & $x, y, z(\mathrm{~mm})$ & Zscore \\
\hline \multicolumn{3}{|l|}{ Rhythm perception ${ }^{a}$} \\
\hline \multicolumn{3}{|l|}{ REPRODUCE } \\
\hline Left V/VI lobule & $-10,-56,-10$ & 4.3 \\
\hline Right V/VI lobule & $12,-54,-12$ & 3.55 \\
\hline Left Crus I & $-40,-60,-38$ & 3.26 \\
\hline Right Crus I/Crus II & $30,-64,-30$ & 3.55 \\
\hline Left lobule VIIB & $-28,-70,-54$ & 3.44 \\
\hline Left inferior olive & $-2,-32,-54$ & 3.07 \\
\hline Right inferior olive & $2-34-56$ & 2.67 \\
\hline \multicolumn{3}{|l|}{ MATCH } \\
\hline Left V/VI lobule & $-20,-78,-20$ & 3.28 \\
\hline \multicolumn{3}{|l|}{ Right V/VI lobule } \\
\hline \multicolumn{3}{|l|}{ Left Crus I } \\
\hline Right Crus I/Crus II & $32,-74,-36$ & 3.75 \\
\hline \multicolumn{3}{|l|}{ Left lobule VIIB } \\
\hline Left inferior olive & $-4,-30,-56$ & 3.51 \\
\hline Right inferior olive & $2-30-56$ & 3.11 \\
\hline \multicolumn{3}{|l|}{ Motor performance of rhythm ${ }^{b}$} \\
\hline \multicolumn{3}{|l|}{ REPRODUCE } \\
\hline Left lobule VI/Crus I & $-34,-60,-34$ & 4.34 \\
\hline Right lobule VI/Crus I & $42,-50,-36$ & 5.02 \\
\hline Left lobule V & $-26,-62,-34$ & 4.2 \\
\hline Right lobule V & $20,-52,-28$ & 4.06 \\
\hline Left Crus I & $-46,-56,-36$ & 4.67 \\
\hline Right Crus I & $44,-58,-36$ & 4.95 \\
\hline Left lobule VIII & $-18,-72,-54$ & 5.1 \\
\hline Right lobule VIII & $20,-68,-54$ & 5.25 \\
\hline R red nucleus & $8,-12,-10$ & 4.33 \\
\hline L red nucleus & $-14,-6,-2$ & 3.85 \\
\hline \multicolumn{3}{|l|}{ SYNCHRONIZE } \\
\hline Left lobule VI/Crus I & $-38,-50,-36$ & 3.56 \\
\hline Right lobule VI/Crus I & $36,-56,-32$ & 4.59 \\
\hline \multicolumn{3}{|l|}{ Left lobule V } \\
\hline \multicolumn{3}{|l|}{ Right lobule V } \\
\hline \multicolumn{3}{|l|}{ Left Crus I } \\
\hline \multicolumn{3}{|l|}{ Right Crus I } \\
\hline Left lobule VIII & $-24,-66,-52$ & 3.61 \\
\hline Right lobule VIII & $14,-70,-50$ & 3.35 \\
\hline Right red nucleus & $10,-14,0$ & 3.01 \\
\hline Left red nucleus & $-12,-18,0$ & 3.30 \\
\hline
\end{tabular}

The cerebellar region nomenclature is according to Schmahmann's atlas (Schmahmann, 1999).

${ }^{a}$ When perceiving complex rhythms compared with isochronous control rhythms in REPRODUCE (perception and reproduction of perceived rhythm) and MATCH (perception and delayed matching) tasks.

${ }^{b}$ When performing complex rhythms compared with isochronous control rhythms in REPRODUCE (reproduction of rhythm from memory) and SYNCHRONIZE (stimulus-guided synchronization) tasks.

metric maps of $t$ statistic for every voxel (transformed to $Z$ statistics) were obtained for each of these four events by subtracting the corresponding events in control conditions using linear contrasts of the parameter estimates. These contrasts were used in the second-level group analysis treating intersubject variability as a random effect and allowing statistical inference at the population level (Penny and Holmes, 2003). A statistical threshold of $Z \geq 3.09$ ( $p \leq 0.001$, uncorrected for multiple comparisons) was used for random-effect results. Homologous activations below the statistical threshold are listed for comparison. The cerebellar atlas and atlas for deep cerebellar nuclei was used for anatomical localization and nomenclature (Schmahmann et al., 1999; Dimitrova et al., 2002).

\section{Results}

\section{Behavioral data}

As expected, compared with control rhythms, complex rhythms showed a longer reaction time (measured from the onset of each of the six stimuli in each rhythm to the onset of motor response) in the SYNCHRONIZE trials [262.7 \pm 25.9 vs $254.8 \pm 33.9 \mathrm{~ms}$ $\left.(\mathrm{SD}) ; F_{(1,783)}=4.36 ; p \leq 0.037\right]$ and a larger asynchrony (differ-
Table 2. Areas specific to perception and motor performance of rhythm

\begin{tabular}{lcc}
\hline Region & $x, y, z$ coordinates $(\mathrm{mm})$ & Zscore \\
\hline Rhythm perception & & \\
Left lobule V/VI & $-10,-58,-8$ & 6.38 \\
Left Crus I & $-30,-72,-44$ & 3.71 \\
Right Crus I & $30,-74,-38$ & 5.49 \\
Left Crus II & $-46,-58,-46$ & 3.74 \\
Right Crus II & $40,-68,-48$ & 3.62 \\
Left lobule VIIB & $-20,-74,-48$ & 3.98 \\
Right inferior olive & $4-32-54$ & 3.96 \\
Left inferior olive & $-2,-32,-54$ & 4.73 \\
Motor performance of rhythm & $-42,-52,-36$ & \\
Left lobule VI & $42,-50,-36$ & 5.9 \\
Right lobule VI & $-22,-68,-54$ & 7.7 \\
Left lobule VIIIB & $14,-72,-52$ & 6.63 \\
Right lobule VIIIB & $6,-76,-40$ & 6.14 \\
Right lobule VIIB (vermis) & $-14,-12,0$ & 3.53 \\
Left red nucleus & $10,-14,0$ & 4.43 \\
Right red nucleus & $6,-56,-16$ & 5.47 \\
Perception and motor performance of rhythm & \\
Right lobule V (vermis) & $-34,-66,-30$ & 4.2 \\
Left Crus I/VI lobule & $-20,-74,-50$ & 4.2 \\
Right Crus II/lobule VIIIB & & 4.31 \\
\hline
\end{tabular}

Data are rhythm perception, motor performance of rhythm, and areas activated in common to perception and moto performance of rhythm regardless of the task performed and without significant interaction between tasks.

ence between interstimulus intervals and the corresponding inter-response intervals in reproduced rhythms) in the REPRODUCE trials $\left[63.29 \pm 111.51\right.$ vs $22.57 \pm 95.07(\mathrm{SD}) \mathrm{ms} ; F_{(1,1414)}$ $=8.57 ; p=0.01]$. Error was $<1 \%$ in the MATCH trials for all rhythms.

\section{fMRI data}

Here, we focus on activations within the cerebellum and brain stem. Please see supplemental material (Tables 1-6, available at www.jneurosci.org) for whole-brain activations. Areas activated during rhythm perception in the REPRODUCE and MATCH trials and during motor performance of rhythm in the REPRODUCE and SYNCHRONIZE trials are listed in Table 1.

Areas specific to rhythm perception were defined as those showing (1) higher response during perception of complex rhythms compared with control rhythms (as shown by subtraction analysis) and (2) significant activation during rhythm perception in both the REPRODUCE and MATCH trials (as shown by conjunction analysis). Multiple areas in the medial and lateral cerebellar cortices and inferior olives bilaterally met both criteria (Table 2, Fig. 1). There was no activation that could be localized to the deep cerebellar nuclei. Figure 2 shows inferior olive activation during perception of rhythm regardless of the task performed and without a significant interaction between tasks.

Areas specific to motor performance of rhythm were similarly defined by two criteria: (1) higher response during performance of complex rhythms compared with control rhythms (i.e., after subtraction of corresponding motor events in control conditions) and (2) significant activation during rhythm performance regardless of the task performed (as shown by conjunction analysis of motor response in the REPRODUCE and SYNCHRONIZE trials). These were in multiple areas in the cerebellar cortex including sensorimotor areas (in the anterior and posterior lobes of the cerebellum bilaterally) corresponding to areas shown in previous fMRI studies of hand movement/somatosensory stimulation (Table 2, Fig. 1) (Bushara et al., 2001; Grodd et al., 2001). Motor performance of rhythm (in both the REPRODUCE and SYNCHRONIZE trials) also activated an area within the brain 
A
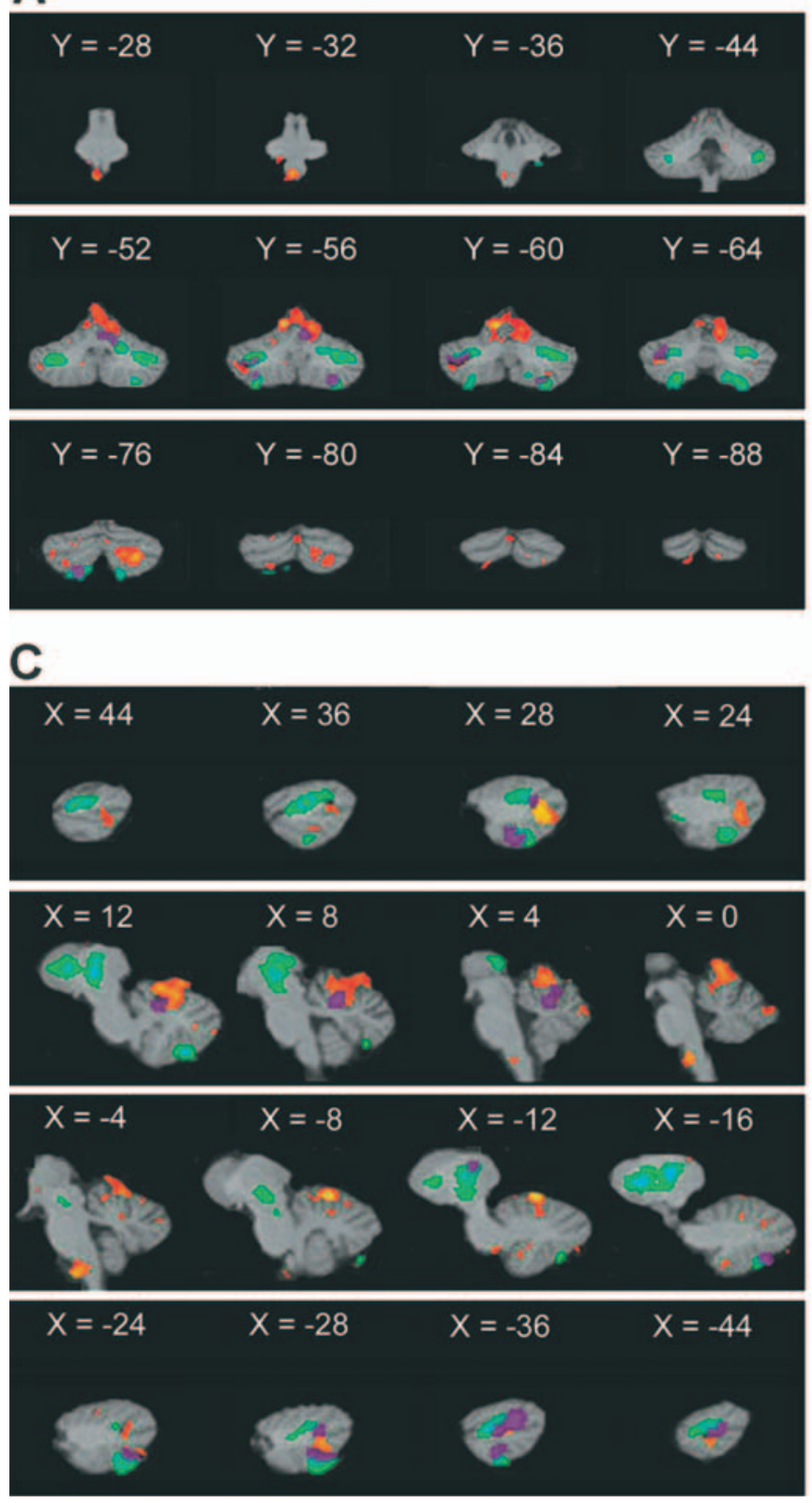

B
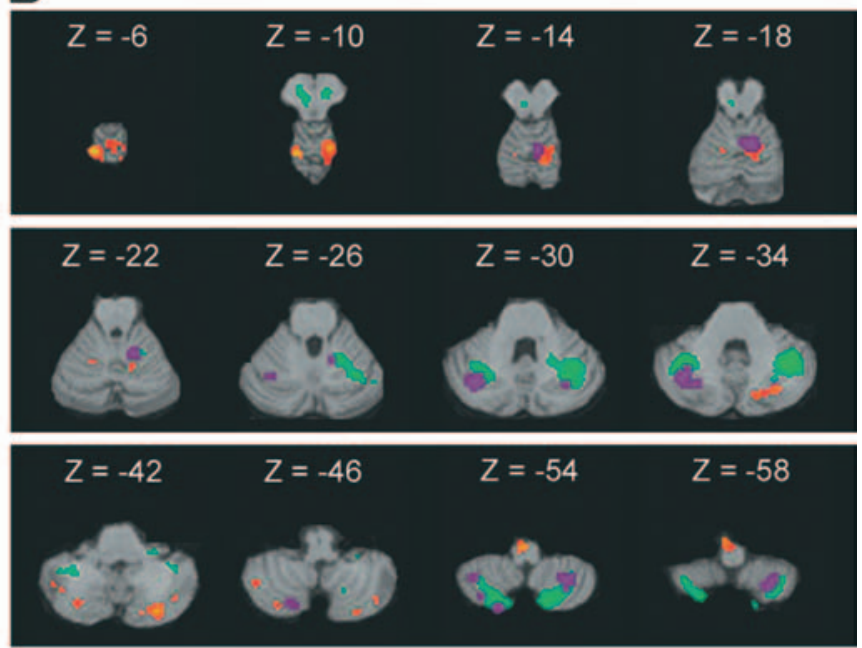

Figure 1. Areas specific to perception and motor performance of rhythm. Statistical parametric maps of event-related activations during rhythm perception regardless of the task performed (perception and delayed matching or perception and reproduction of perceived rhythm) (red/yellow), motor performance of rhythm regardless of the task (synchronize or reproduce tasks) (green), and areas activated in common to perception and motor performance of rhythm without significant interaction between tasks (purple) are shown. Activations are shown on coronal $(\boldsymbol{A})$, axial $(\boldsymbol{B})$, and sagittal $(\boldsymbol{C})$ templates. Stereotaxic coordinates are as follows (in millimeters relative to the anterior commissure): $X$, right $(+)$ and left $(-)$; $Y$, anterior $(+)$ and posterior $(-) ; Z$, superior $(+)$ and inferior (-).

stem in the region of the midbrain/red nucleus. Again, there was no activation that could be localized to the deep cerebellar nuclei. The inferior olives showed no activation to motor responses during either the REPRODUCE or SYNCHRONIZE tasks. This was also true even without subtraction of the corresponding control conditions (supplemental Tables 5, 6, available at www.jneurosci. org as supplemental material). Therefore, lack of inferior olive activity during motor tasks cannot be attributed to subtracting out of the motor-related responses during performance of control rhythms.

The specificity of inferior olive activation to rhythm perception was further tested using direct subtraction of motorresponse maps (motor response in the REPRODUCE and SYN-
CHRONIZE trials) from rhythm-perception maps (activation to rhythm perception events in common to the MATCH and REPRODUCE trials). This showed significant inferior olive activation after subtraction of the response to motor events in the REPRODUCE trial $(x, y, z$ coordinates and $Z$ score at peak inferior olive activity: $2,-28,-58, z=3.52$; and $-4,-32,-60, z=$ 2.78 ) and after subtraction of the response to motor events in the SYNCHRONIZE trial $(-4,-28,-56, z=2.87)$. There was no significant interaction between SYNCHRONIZE and REPRODUCE tasks within the inferior olive.

Finally, we identified areas activated in common to both perception and motor performance of rhythm regardless of the task performed and without significant interaction between tasks. 


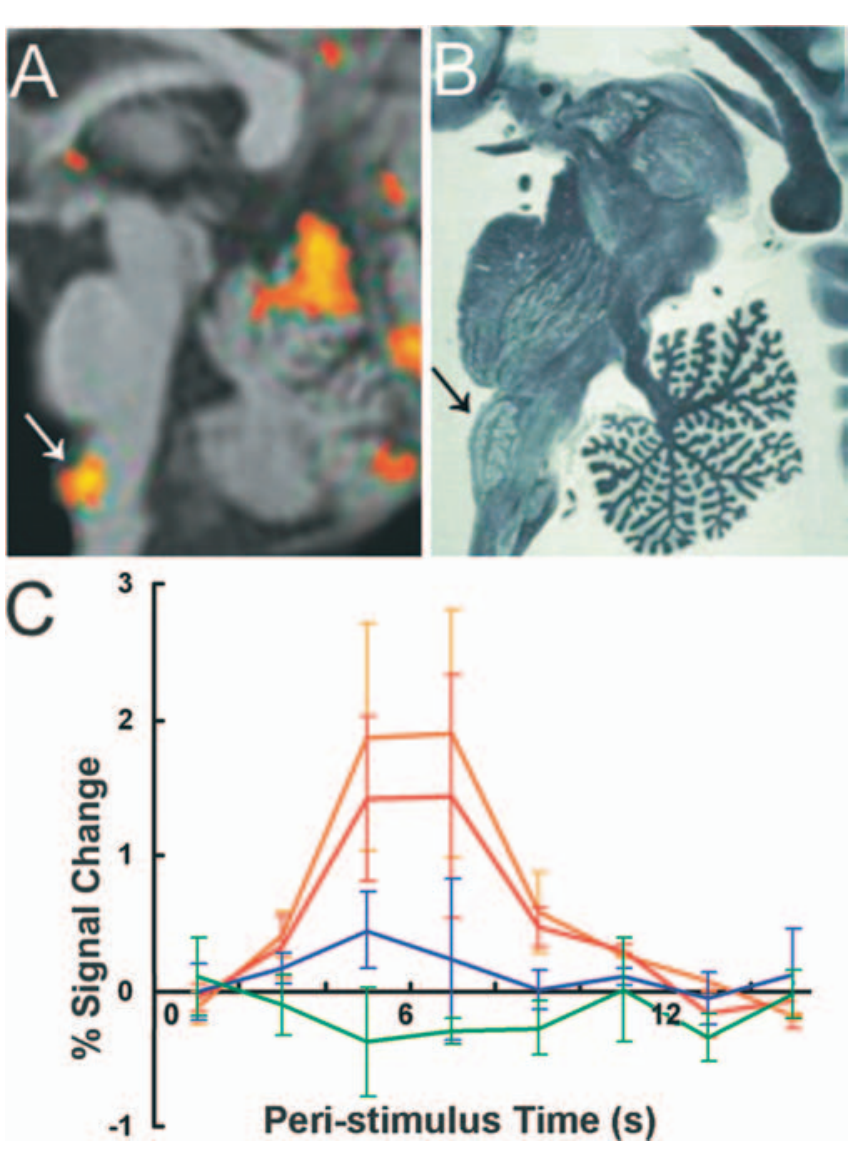

Figure 2. $\quad \boldsymbol{A}$, Event-related activation of the inferior olive (white arrow) time locked to the perception of temporal sequence. $\boldsymbol{B}$, Corresponding sagittal anatomical section showing the inferior olive (black arrow). $\boldsymbol{C}$, Time course of the average hemodynamic response at inferior olive peak activation $(-2,-32,-54)$ time locked to perception in REPRODUCE trials (red), perception in MATCH trials (orange), motor reproduction from memory (green), and stimulusguided motor response synchronized to the temporal sequence (blue). Error bars indicate SD. Peristimulus time is in seconds.

These were in multiple cerebellar areas but not the inferior olive (Table 2, Fig. 1).

\section{Discussion}

Our results show activation of multiple areas within the cerebellar cortex during both perception and motor performance of temporal sequences. Neither motor performance nor motor preparation was a prerequisite for cerebellar activity during timing tasks. These results support the view that the cerebellum contributes to motor and nonmotor timing and are in general agreement with the timing hypothesis and other theories advocating a basic cerebellar function that is required for both motor and nonmotor activity (Ito, 1993; Ivry, 1997; Brown and Bower, 2002; Schmahmann, 2004).

An important finding of this study is the activation of the inferior olive during perception of complex temporal sequences, which provides new evidence linking inferior olive activity to timing behavior. This has not been shown in previous functional imaging studies of timing (Rao et al., 1997, 2001; Schubotz et al., 2000; Ramnani and Passingham, 2001; Dhamala, 2003). Although the inferior olive neurons have been shown to respond robustly to visual and somatosensory stimuli (Bloedel and Ebner, 1984; Simpson, 1984), the activation of the inferior olive in the current study was specific to the perception complex rhythms and cannot be attributed to visual stimulation alone (Bloedel and
Ebner, 1984; Simpson, 1984). Visual stimulation in control conditions was identical to that in rhythm perception tasks, which differed only in the temporal structure of the sequence of stimuli. Unlike the temporal sequences used in the current study, which contained multiple time intervals, previous imaging studies of timing mostly applied a single time interval to be perceived and encoded, which may explain the lack of inferior olive activation in these studies (Rao et al., 1997, 2001).

Our results showed no activation of the inferior olive during motor performance of rhythms in either reproduction or synchronization tasks even without subtraction of the corresponding control conditions. Because the synchronization task required both perception and motor performance of temporal sequences, the "suppression" of the inferior olive response to the perception component of the task can conceivably be attributed to the concurrent motor activity. This interpretation would be most consistent with electrophysiological studies showing that the responsiveness of the olivary neurons to sensory input is mostly decreased during expected self-produced movement (Gellman et al., 1985; Horn et al., 1996; Apps et al., 1997; Gibson et al., 2002). This may further explain the failure to demonstrate inferior olive activation during timing tasks in previous functional imaging studies that mostly used paradigms requiring motor preparation or motor response (Rao et al., 1997; Schubotz et al., 2000; Ramnani and Passingham, 2001; Dhamala et al., 2003).

Another possible explanation for the lack of inferior olive activation during motor timing tasks is that motor timing may differentially involve the medial and/or dorsal accessory olives (MAO and DAO), whereas perceptual timing involves the principal olive (PO). It has been postulated that the intermediate cerebellum through its direct connections with the motor system mediates the motor aspects of timing, whereas the lateral cerebellar hemispheres and their output nuclei (the dentate nuclei) mediate the cognitive or perceptual aspects of timing through its connections with the frontoparietal association cortex (Middleton and Strick, 1994). The DAO and MAO receive projections from the intermediate cerebellum, whereas the $\mathrm{PO}$ receives projections (via the dentate nucleus) from the lateral cerebellar hemispheres (Kalil, 1979). Compared with the PO, the MAO and $\mathrm{DAO}$ are much smaller in size in humans, and their activation is less likely to be detected with current fMRI methods. Moreover, fMRI methods may not detect motor-related activity if represented with population rather than frequency coding as proposed for the inferior olivary neurons (Welsh, 2002). Therefore, the lack of inferior olive activity during motor timing tasks in the current study and previous imaging studies does not necessarily rule out a role for the inferior olive in motor timing or timing error correction during movement. Furthermore, the current study does not address motor timing processes related to motor learning and classical conditioning that have also been proposed as a function of the olivo-cerebellar system (Mauk et al., 1986; De Zeeuw et al., 1998; Koekkoek et al., 2003). Nonetheless, our results provide evidence relating the inferior olive activity to the processing of temporal information regardless of motor activity. Based on our data, we propose that the primary role of the inferior olive and the climbing fiber system in timing is to mediate the encoding of temporal information independent of motor behavior.

\section{References}

Allen G, Buxton RB, Wong EC, Courchesne E (1997) Attentional activation of the cerebellum independent of motor involvement. Science 275:1940-1943.

Apps R, Atkins MJ, Garwicz M (1997) Gating of cutaneous input to cerebel- 
lar climbing fibres during a reaching task in the cat. J Physiol (Lond) 502:203-214.

Armstrong DM (1974) Functional significance of connections of the inferior olive. Physiol Rev 54:358-417.

Bloedel JR, Ebner TJ (1984) Rhythmic discharge of climbing fibre afferents in response to natural peripheral stimuli in the cat. J Physiol (Lond) 352:129-146.

Braitenberg V (1967) Is the cerebellar cortex a biological clock in the millisecond range? Prog Brain Res 25:334-346.

Brown IE, Bower JM (2002) The influence of somatosensory cortex on climbing fiber responses in the lateral hemispheres of the rat cerebellum after peripheral tactile stimulation. J Neurosci 22:6819-6829.

Bushara KO, Wheat JM, Khan A, Mock BJ, Turski PA, Sorenson J, Brooks BR (2001) Multiple tactile maps in the human cerebellum. NeuroReport 12:2483-2486.

Dale AM (1999) Optimal experimental design for event-related fMRI. Hum Brain Mapp 8:109-114.

De Zeeuw CI, Simpson JI, Hoogenraad CC, Galjart N, Koekkoek SK, Ruigrok TJ (1998) Microcircuitry and function of the inferior olive. Trends Neurosci 21:391-400.

Dhamala M, Pagnoni G, Wiesenfeld K, Zink CF, Martin M, Berns GS (2003) Neural correlates of the complexity of rhythmic finger tapping. NeuroImage 20:918-926.

Dimitrova A, Weber J, Redies C, Kindsvater K, Maschke M, Kolb FP, Forsting M, Diener HC, Timmann D (2002) MRI atlas of the human cerebellar nuclei. NeuroImage 17:240-255.

Essens PJ, Povel DJ (1985) Metrical and nonmetrical representations of temporal patterns. Percept Psychophys 37:1-7.

Fraisse P (1974) Psychologie du rhythme. Paris: Presses Universitaires de France.

Gao JH, Parsons LM, Bower JM, Xiong J, Li J, Fox PT (1996) Cerebellum implicated in sensory acquisition and discrimination rather than motor control. Science 272:545-547.

Gellman R, Gibson AR, Houk JC (1985) Inferior olivary neurons in the awake cat: detection of contact and passive body displacement. J Neurophysiol 54:40-60.

Gibson AR, Horn KM, Pong M (2002) Inhibitory control of olivary discharge. Ann NY Acad Sci 978:219-231.

Gibson AR, Horn KM, Pong M (2004) Activation of climbing fibers. Cerebellum 3:212-221.

Grodd W, Hulsmann E, Lotze M, Wildgruber D, Erb M (2001) Sensorimotor mapping of the human cerebellum: fMRI evidence of somatotopic organization. Hum Brain Mapp 13:55-73.

Harrington DL, Lee RR, Boyd LA, Rapcsak SZ, Knight RT (2004a) Does the representation of time depend on the cerebellum? Effect of cerebellar stroke. Brain 127:561-574.

Harrington DL, Boyd LA, Mayer AR, Sheltraw DM, Lee RR, Huang M, Rao SM (2004b) Neural representation of interval encoding and decision making. Brain Res Cogn Brain Res 21:193-205.

Horn KM, Van Kan PL, Gibson AR (1996) Reduction of rostral dorsal accessory olive responses during reaching. J Neurophysiol 76:4140-4151.

Ito M (1993) Movement and thought: identical control mechanisms by the cerebellum. Trends Neurosci 16:448-450; discussion 453-444.

Ivry R (1997) Cerebellar timing systems. Int Rev Neurobiol 41:555-573.

Ivry RB, Keele SW, Diener HC (1988) Dissociation of the lateral and medial cerebellum in movement timing and movement execution. Exp Brain Res 73:167-180.

Kalil K (1979) Projections of the cerebellar and dorsal column nuclei upon the inferior olive in the rhesus monkey: an autoradiographic study. J Comp Neurol 188:43-62.

Keating JG, Thach WT (1995) Nonclock behavior of inferior olive neurons: interspike interval of Purkinje cell complex spike discharge in the awake behaving monkey is random. J Neurophysiol 73:1329-1340.

Keele SW, Ivry R (1990) Does the cerebellum provide a common computa- tion for diverse tasks? A timing hypothesis. Ann NY Acad Sci 608:179-207.

Kim SG, Ugurbil K, Strick PL (1994) Activation of a cerebellar output nucleus during cognitive processing. Science 265:949-951.

Koekkoek SK, Hulscher HC, Dortland BR, Hensbroek RA, Elgersma Y, Ruigrok TJ, De Zeeuw CI (2003) Cerebellar LTD and learningdependent timing of conditioned eyelid responses. Science 301:1736-1739.

Leiner HC, Leiner AL, Dow RS (1993) Cognitive and language functions of the human cerebellum. Trends Neurosci 16:444-447.

Llinas R, Sasaki K (1989) The functional organization of the olivo-cerebellar system as examined by multiple Purkinje cell recordings. Eur J Neurosci 1:587-602.

Mauk MD, SteinmetzJE, Thompson RF (1986) Classical conditioning using stimulation of the inferior olive as the unconditioned stimulus. Proc Natl Acad Sci USA 83:5349-5353.

Middleton FA, Strick PL (1994) Anatomical evidence for cerebellar and basal ganglia involvement in higher cognitive function. Science 266:458-461.

Oscarsson O (1980) Functional organization of olivary projection to the cerebellar anterior lobe. In: The inferior olivary nucleus: anatomy and physiology (Courville J, De Montigny C, Lamarre Y, Universitâe de Montrâeal Centre de Recherche en Sciences Neurologiques, eds), pp xi, 396p. New York: Raven.

Penny W, Holmes A (2003) Random effect analysis. In: Human brain function (Frackowiak RSJFK, Frith CD, Dolan R, Price CJ, Zeki S, Ashburner J, Penny WD, eds), pp 843-850. New York: Academic.

Petersen SE, Fox PT, Posner MI, Mintun M, Raichle ME (1988) Positron emission tomographic studies of the cortical anatomy of single-word processing. Nature 331:585-589.

Price CJ, Friston KJ (1997) Cognitive conjunction: a new approach to brain activation experiments. NeuroImage 5:261-270.

Ramnani N, Passingham RE (2001) Changes in the human brain during rhythm learning. J Cogn Neurosci 13:952-966.

Rao SM, Harrington DL, Haaland KY, Bobholz JA, Cox RW, Binder JR (1997) Distributed neural systems underlying the timing of movements. J Neurosci 17:5528-5535.

Rao SM, Mayer AR, Harrington DL (2001) The evolution of brain activation during temporal processing. Nat Neurosci 4:317-323.

Schmahmann JD (2004) Disorders of the cerebellum: ataxia, dysmetria of thought, and the cerebellar cognitive affective syndrome. J Neuropsychiatry Clin Neurosci 16:367-378.

Schmahmann JD, Sherman JC (1998) The cerebellar cognitive affective syndrome. Brain 121:561-579.

Schmahmann JD, Doyon J, McDonald D, Holmes C, Lavoie K, Hurwitz AS, Kabani N, Toga A, Evans A, Petrides M (1999) Three-dimensional MRI atlas of the human cerebellum in proportional stereotaxic space. NeuroImage 10:233-260.

Schubotz RI, Friederici AD, von Cramon DY (2000) Time perception and motor timing: a common cortical and subcortical basis revealed by fMRI. NeuroImage 11:1-12.

Simpson JI (1984) The accessory optic system. Annu Rev Neurosci 7:13-41.

Thach WT (1968) Discharge of Purkinje and cerebellar nuclear neurons during rapidly alternating arm movements in the monkey. J Neurophysiol 31:785-797.

Thach WT (1997) Context-response linkage. Int Rev Neurobiol 41: 599-611.

Welsh JP (2002) Functional significance of climbing-fiber synchrony: a population coding and behavioral analysis. Ann NY Acad Sci 978:188-204.

Welsh JP, Lang EJ, Suglhara I, Llinas R (1995) Dynamic organization of motor control within the olivocerebellar system. Nature 374:453-457.

Welsh JP, Ahn ES, Placantonakis DG (2005) Is autism due to brain desynchronization? Int J Dev Neurosci 23:253-263. 